\title{
Quantum correction for electron transfer rates. Comparison of polarizable versus nonpolarizable descriptions of solvent
}

\author{
Xueyu Song and R. A. Marcus \\ Arthur Amos Noyes Laboratory of Chemical Physics, ${ }^{a)}$ 127-72, California Institute of Technology, \\ Pasadena, California 91125
}

(Received 29 July 1993; accepted 13 August 1993)

\begin{abstract}
The electron transfer rate constant is treated using the spin-boson Hamiltonian model. The spectral density is related to the experimentally accessible data on the dielectric dispersion of the solvent, using a dielectric continuum approximation. On this basis the quantum correction for the ferrous-ferric electron transfer rate is found to be a factor 9.6. This value is smaller than the corresponding result (36) of Chandler and co-workers in their pioneering quantum simulation using a molecular model of the system [J. S. Bader, R. A. Kuharski, and D. Chandler, J. Chem. Phys. 93, 230 (1990)]. The likely reason for the difference lies in use of a rigid water molecular model in the simulation, since we find that other models for water in the literature which neglect the electronic and vibrational polarizability also give a large quantum effect. Such models are shown to overestimate the dielectric dispersion in one part of the quantum mechanically important region and to underestimate it in another part. It will be useful to explore a polarizable molecular model which reproduces the experimental dielectric response over the relevant part of the frequency spectrum.
\end{abstract}

\section{INTRODUCTION}

Electron transfer reactions are among the most fundamental chemical processes. ${ }^{1-3}$ As a prototypical model system, the $\mathrm{Fe}^{+2} \rightleftharpoons \mathrm{Fe}^{+3}$ electron exchange in water has been actively studied. ${ }^{4,5}$ Recently, Chandler and co-workers ${ }^{6,7}$ used a molecular model to study this process by quantum and classical simulation methods. Their results have shed an illuminating light on this system.

By classical and quantum simulation methods they observed the parabolic behavior of the free energy surface with respect to the solvent polarization coordinate, a behavior which plays the important role in the theory developed by one of us. ${ }^{1}$ They also studied the quantum correction for the electron transfer rate constant. The full quantum Monte Carlo simulation gave a quantum correction factor for the rate of about 65 for water. ${ }^{6}$ Under a harmonic approximation their quantum correction was about 36, which is still substantially larger than the traditional estimate ${ }^{5}$ of a factor of about 7 .

In the present paper an expression for the nonadiabatic rate constant (the Golden Rule rate expression) is used in which the rate is expressed in terms of the spectral density (the dielectric response) of the system. The spectral density is then obtained from experimental data, for fixed position of the reactants, using the dielectric continuum approximation and the harmonic approximation for the inner shell of ion-water complex breathing modes. For the electron transfer rate constant for the aqueous ferrous-ferric system it is found that under the above approximation our estimation of the quantum correction factor is a factor of 9.6, which is smaller than the above result of 36 for the harmonic case. Other computer simulation models of water which also neglect the vibrational and the electronic polarization are also considered. We again find a large quantum effect for the rate constant of the model water solvent and find that these models overestimate the dielectric response in one region important for the quantum correction and underestimate it in another.

This paper is organized as follows: In Sec. II the theoretical basis of this paper is discussed. The nonadiabatic rate constant is expressed there in terms of the spectral density of the system. The relations between the spectral density and the experimental data are given. In Sec. III the calculation details are presented and the role of the electronic polarizability in electron transfer is discussed. The paper concludes with some remarks.

\section{THEORY}

\section{A. Introduction}

In this section a brief discussion of the nonadiabatic rate constant expression is given. Then, the relation between the spectral density and the experimentally accessible data, which forms the basis of the calculations, is presented.

In electron transfer reactions the reactant and product electronic states can usually be approximated as a two electronic-state system. If the solvent and the nuclear motion of the reactants and products are described as a harmonic bath, the electron transfer can be viewed as an electron jump between the two states modulated by a harmonic bath. This kind of system has been described by the spinboson Hamiltonian ${ }^{6,8,9}$

$$
\begin{aligned}
H= & \frac{H_{A B}}{2} \sigma_{x}+\frac{\epsilon}{2} \sigma_{z}+\frac{\sigma_{z}}{2} \sum_{i=1}^{N} 2 c_{i} y_{i} \\
& +\sum_{i=1}^{N}\left(\frac{p_{i}^{2}}{2 m_{i}}+\frac{m_{i} \omega_{i}^{2} y_{i}^{2}}{2}\right)
\end{aligned}
$$

a) Contribution No. 8833 . 
where $H_{A B} / 2$ is the electronic matrix element which couples the reactants' state $|-\rangle$ and the products' state $|+\rangle$. The $y_{i}$ is a polarization coordinate, $p_{i}$ is the canonically conjugate momentum, and $\epsilon$ is the driving force of the reaction (i.e., $\left.-\Delta G^{0}\right), \sigma_{z}$ and $\sigma_{x}$ are Pauli matrices in the two-state $(|+\rangle$ and $|-\rangle)$ representation. A harmonic bath having the same frequencies for the reactants' and the products' electronic states is assumed in this Hamiltonian. In such an approximation any changes in metal-ligand frequencies accompanying the electron transfer are replaced by a particular symmetric combination. ${ }^{5}$

For the nonadiabatic case, the electron transfer rate constant is given by the following Golden Rule formula: ${ }^{2,3}$

$$
\begin{aligned}
k= & \frac{2 \pi}{\hbar Z_{b}}\left|\frac{H_{A B}}{2}\right|^{2} \sum_{i} \sum_{f} \exp \left(-\beta E_{i}^{0}\right)\left|\left\langle\chi_{i}^{0} \mid \chi_{f}^{0}\right\rangle\right|^{2} \\
& \times \delta\left(E_{f}^{0}-E_{i}^{0}+\epsilon\right),
\end{aligned}
$$

where $i$ and $f$ specify the quantum numbers of the reactant and the product system, $E_{i}^{0}$ and $E_{f}^{0}$ are the energy levels of their systems, $\chi_{i}^{0}$ and $\chi_{f}^{0}$ are the corresponding wave functions, and $Z_{b}$ is the partition function of the reactant system.

Upon using the usual $\delta$-function expression

$$
\begin{aligned}
& \delta\left(E_{f}^{0}-E_{i}^{0}+\epsilon\right) . \\
& \quad=\frac{\beta}{2 \pi} \int_{-\infty}^{\infty} \exp \left[-i\left(E_{f}^{0}-E_{i}^{0}+\epsilon\right) \beta R\right] d R
\end{aligned}
$$

and the overlap integral of the wave functions assumed to be harmonic, the following formula has been obtained:,3

$$
\begin{aligned}
k= & \frac{\beta}{\hbar}\left|\frac{H_{A B}}{2}\right|^{2} \int_{-\infty}^{+\infty} d R \exp \left[-\left(\frac{\beta}{2}+i \beta R\right) \epsilon\right. \\
& \left.-\frac{2}{\pi \hbar} \int_{0}^{\infty} d \omega \frac{J(\omega)}{\omega^{2}} \frac{\cosh (\beta \hbar \omega / 2)-\cosh (i R \beta \hbar \omega)}{\sinh (\beta \hbar \omega / 2)}\right],
\end{aligned}
$$

where $J(\omega)$ is spectral density of the system

$$
J(\omega)=\sum_{i=1}^{N} \pi \delta\left(\omega-\omega_{i}\right) \frac{c_{i}^{2}}{m_{i} \omega_{i}}
$$

This well-known quantum rate constant expression of electron transfer in the nonadiabatic case was first derived by Soviet scientists. ${ }^{2,3}$ Later Chandler and co-workers ${ }^{6}$ rederived this result within a spin-boson Hamiltonian description by the Golden Rule. Recently, ${ }^{8}$ Song and Stuchebrukhov gave a general description of electron transfer reactions using the spin-boson Hamiltonian, in which the above formula appears as a special case of a more general one. A key assumption in the above formula is the use of a harmonic approximation for the bath modes. ${ }^{2}$ Equation (2.4) is the fundamental result of nonadiabatic quantum electron transfer theory within the harmonic approximation. Another approach, in which only a linear response approximation is used for the solvent bath, ${ }^{2(c)}$ leads to the same result, where the $J(\omega)$ is expressed, as given later, in terms of the dielectric response function.
TABLE I. The quantum correction of aqueous ferrous-ferric system $(T=298 \mathrm{~K})$.

\begin{tabular}{lcccc}
\hline \hline & $\begin{array}{c}\text { Saddle-point approximation } \\
\text { Inner part }\end{array}$ & Outer part & Total & Ratio \\
\hline Quantum exponential & -12.97 & -10.84 & -23.81 & \\
& & & & 8.4 \\
Classical exponential & -14.11 & -11.86 & -25.97 & \\
Quantum prefactor & & & $1.65 \times 10^{11}$ & \\
Classical prefactor & & $1.48 \times 10^{11}$ & 1.1 \\
Quantum rate $\left(\mathrm{s}^{-1}\right)$ & & 7.53 & \\
Classical rate $\left(\mathrm{s}^{-1}\right)$ & & $0.68\left(36^{c}\right)$ &
\end{tabular}

Full calculation $^{\mathrm{b}}$

Quantum rate $\left(\mathrm{s}^{-1}\right)$

Classical rate $\left(\mathrm{s}^{-1}\right)$

0.78

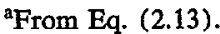

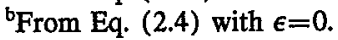

${ }^{\mathrm{c}}$ From Ref. 6.

For the ferrous-ferric system $\epsilon$ is zero. The saddlepoint of $R$ is zero in this case. The rate constant can be expressed in the following simple form using the saddlepoint approximation, ${ }^{5}$

$$
\begin{aligned}
k \approx & \frac{2 \pi}{\hbar}\left|\frac{H_{A B}}{2}\right|^{2}\left[4 \hbar \int_{0}^{\infty} d \omega J(\omega) \cosh \left(\frac{\beta \hbar \omega}{2}\right)\right]^{-1 / 2} \\
& \times \exp \left[-\frac{2}{\pi \hbar} \int_{0}^{\infty} d \omega \frac{J(\omega)}{\omega^{2}} \tanh \left(\frac{\beta \hbar \omega}{4}\right)\right] .
\end{aligned}
$$

In the classical approximation for bath $(\beta \hbar \omega<1)$ Eq. (2.6) reduces to the usual classical nonadiabatic expression for the rate of electron transfer in symmetric reactions. A test of the saddlepoint approximation, by a comparison of Eqs. (2.6) and (2.4), is given later in Table I.

In order to calculate the electron transfer rate constant for the actual system the explicit form of spectral density $J(\omega)$ is needed. There are several ways of obtaining this quantity. For example, Bader et al. ${ }^{6,7}$ calculate it by a quantum Monte Carlo simulation using a microscopic model of aqueous ferrous-ferric system. Another way is to relate this quantity to some phenomenological experimentally measurable variable. ${ }^{3}$ In the present paper the latter approach is used; the bath is divided into an inner part [the first coordination shell, consisting of $\mathrm{Fe}\left(\mathrm{H}_{2} \mathrm{O}\right)_{6}^{+2}-$ $\mathrm{Fe}\left(\mathrm{H}_{2} \mathrm{O}\right)_{6}^{+3}$ subsystem] contributing an amount $J_{i}(\omega)$ to the spectral density, and an outer part, namely the rest of the water solvent interacting with the hexacoordinated aqueous $\mathrm{Fe}^{+2}$ and $\mathrm{Fe}^{+3}$ ions, treated as spheres. The outer contribution $J_{0}(\omega)$ to the spectral density is then related to experimental data on the dielectric response of the solvent. Thus we have

$$
J(\omega)=J_{0}(\omega)+J_{i}(\omega) .
$$


The electron transfer rate constant is then calculated.

\section{B. The spectral density of the outer contribution}

Phenomenologically, the outer contribution can be treated using a dielectric continuum interacting with the ionic charge distribution. ${ }^{3}$ The response of the dielectric to the electric field is defined by the dielectric permittivity function $\epsilon(\omega)$, which is experimentally available. If the polar medium is treated as a collection of harmonic oscillators characterizing the dielectric polarization, the changes in electric field of the ions, where the products are compared with the reactants, shift the equilibrium positions of oscillators describing the polarization. According to Eq. (3.87) in Ref. 3, the shift of the dielectric polarization oscillator of the medium $q_{i 0}$ is related to the dielectric constant in the following way, neglecting spatial dispersion:

$$
\frac{1}{2} \sum_{i} \hbar \omega_{i} q_{i 0}^{2}=\frac{\int d \mathbf{r}\left|D^{f}-D^{i}\right|^{2}}{4 \pi^{2}} \int_{0}^{\infty} \frac{d \omega}{\omega} \frac{\operatorname{Im} \epsilon(\omega)}{|\epsilon(\omega)|^{2}},
$$

where $\epsilon(\omega)$ is the dielectric constant as a function of frequency, the imaginary part of it being related to the absorption of the medium, $D^{f}$ and $D^{i}$ are the dielectric displacement vectors of the products' and the reactants' forms of the ions. For two ionic spheres with radii $a_{1}$ and $a_{2}$ and separated by a center-to-center distance $R$ ( $\Delta e$ is the charge difference) we have, ${ }^{1,17}$ neglecting dielectric image effects,

$$
\frac{1}{8 \pi} \int d \mathbf{r}\left|D^{f}-D^{i}\right|^{2}=\left(\frac{1}{2 a_{1}}+\frac{1}{2 a_{2}}-\frac{1}{R}\right)(\Delta e)^{2} .
$$

The left-hand side of Eq. (2.8), $\lambda_{0}$, represents the classical form of the outer part of the reorganization energy of the system. For the spin-boson model this reorganization energy can be written as ${ }^{8}$

$$
\lambda_{0}=\sum_{i} \frac{2 c_{i}^{2}}{m_{i} \omega_{i}^{2}}=\int_{0}^{\infty} \frac{2}{\pi} \frac{d \omega}{\omega} J_{0}(\omega) .
$$

The same formula relating $\lambda_{i}$ to $J_{i}(\omega)$ also applies. Comparing Eqs. (2.8) and (2.10), the outer contribution $J_{0}(\omega)$ to the spectral density can be written as

$$
J_{0}(\omega)=\frac{\int d \mathbf{r}\left|D^{f}-D^{i}\right|^{2}}{8 \pi} \frac{\operatorname{Im} \epsilon(\omega)}{|\epsilon(\omega)|^{2}} .
$$

Equation (2.4), with $J$ given by Eq. (2.11), was first obtained by a different method by Ovchinnikov and Ovchinnikova. ${ }^{2(c)}$ They showed that above result is valid under linear response theory. A more elaborate description, based on $\epsilon(k, \omega)$ could be deduced from the results given in Ref. 3, $\mathbf{k}$ being the wave vector. However, the relevant experimental data for $\epsilon(\mathbf{k}, \omega)$ do not appear to be available.

\section{The spectral density of the inner-shell contribution}

The inner part of the spectral density $I_{i}(\omega)$, i.e., the contribution from the inner-shell, is relatively simple. The main contribution for electron transfer comes from the two shifted symmetric breathing modes (they are normal modes) of the reactant pair, $\mathrm{Fe}\left(\mathrm{H}_{2} \mathrm{O}\right)_{6}^{+2}-\mathrm{Fe}\left(\mathrm{H}_{2} \mathrm{O}\right)_{6}^{+3}$, and the product pair, $\mathrm{Fe}\left(\mathrm{H}_{2} \mathrm{O}\right)_{6}^{+3}-\mathrm{Fe}\left(\mathrm{H}_{2} \mathrm{O}\right)_{6}^{+2}{ }^{10,11}$ The equilibrium shift of harmonic coordinates is the equilibrium bond length change $d_{0}$ from $\mathrm{Fe}\left(\mathrm{H}_{2} \mathrm{O}\right)_{6}^{+2}$ to $\mathrm{Fe}\left(\mathrm{H}_{2} \mathrm{O}\right)_{6}^{+3}$, the breathing mode frequency is $\omega_{1}$ for $\mathrm{Fe}\left(\mathrm{H}_{2} \mathrm{O}\right)_{6}^{+2}$ and $\omega_{2}$ for $\mathrm{Fe}\left(\mathrm{H}_{2} \mathrm{O}\right)_{6}^{+3}$, and the constant frequency $\omega_{0}$ used in the Hamiltonian can be expressed approximately ${ }^{1,5}$ by $\omega_{0}^{2}=2 \omega_{1}^{2} \omega_{2}^{2} /\left(\omega_{1}^{2}+\omega_{2}^{2}\right) ; m$ in Eq. (2.5) for $J_{i}$ is the mass for a ligand molecule in a symmetric breathing mode. Thus, the spectral density of the inner contribution can be written as

$$
J_{i}(\omega)=(\pi / 2) \omega \delta\left(\omega-\omega_{0}\right) \lambda_{i},
$$

where

$$
\lambda_{i}=6 m \omega_{0}^{2} d_{0}^{2}
$$

using the fact that there are six ligands for the symmetric breathing mode in $\mathrm{Fe}\left(\mathrm{H}_{2} \mathrm{O}\right)_{6}^{+2}$ and in $\mathrm{Fe}\left(\mathrm{H}_{2} \mathrm{O}\right)_{6}^{+3}$.

\section{Rate constant result}

The calculation of the electron transfer rate constant can be made by direct numerical integration of Eq. (2.4), using Eqs. (2.7), (2.11), (2.12), and the spectral density obtained from the experimental data. Or the saddle-point approximation can be used, so that a somewhat more transparent picture can be obtained. In this case Eq. (2.6) can be written in the following form:

$$
\begin{aligned}
k \approx & \frac{2 \pi}{\hbar}\left|\frac{H_{A B}}{2}\right|^{2}\left[4 \int_{0}^{\infty} d(\ln \omega) J_{0}(\omega) \hbar \omega \cosh \left(\frac{\beta \hbar \omega}{2}\right)\right. \\
& \left.+2 \pi \hbar \omega_{0} \lambda_{i} \cosh \left(\frac{\beta \hbar \omega_{0}}{2}\right)\right]^{-1 / 2} \\
& \times \exp \left[-\int_{0}^{\infty} d(\ln \omega) \frac{2 J_{0}(\omega)}{\pi \hbar \omega} \tanh \left(\frac{\beta \hbar \omega}{4}\right)\right. \\
& \left.-\frac{\lambda_{i}}{\hbar \omega_{0}} \tanh \left(\frac{\beta \hbar \omega_{0}}{4}\right)\right],
\end{aligned}
$$

where $J_{0}(\omega)$ is given by Eq. (2.11) and $\lambda_{i}$ is given by Eq. (2.12b). Because of the wide range of $\omega$ 's which contribute to the integrand from the outer part it was convenient to introduce $\ln \omega$ as the integration variable in $\mathrm{Eq}$. (2.13). Equations (2.4) [with Eqs. (2.7), (2.11), and (2.12)] and Eq. (2.13) represent the starting point for the present calculation.

\section{CALCULATIONS AND DISCUSSION}

In this section the quantum and classical rate constants are calculated from the experimental data for the present model. From the above formulas, a key step is to use the experimentally observed complex-valued dielectric constant of solvent as a function of frequency. In general, there exist two broad regions of absorption in water, the Debye region (or orientational region) and the resonance region. In the Debye region, the Debye formula can be used to 


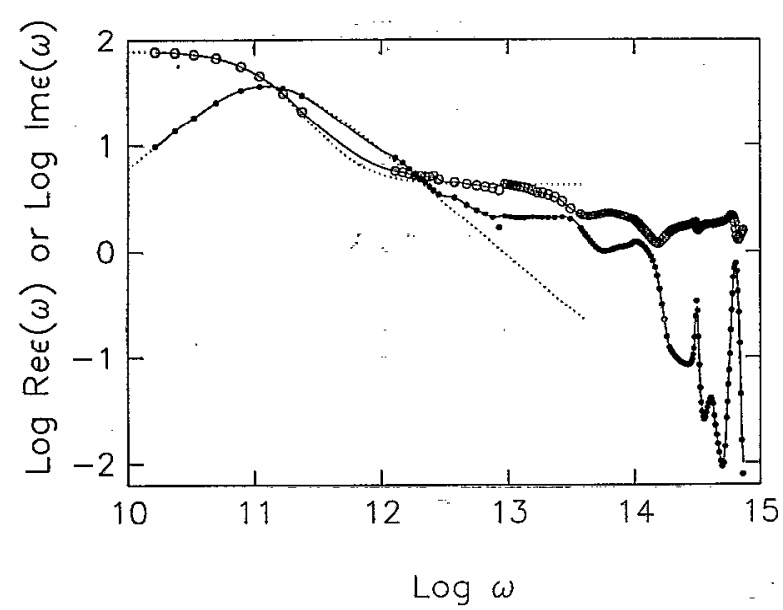

FIG. 1. The experimental data (Refs. 12-15) and the empirical fit. $\bigcirc$ denotes the real part of the experimental dielectric constant; the imaginary part. The solid line is a cubic spline interpolation and the dotted line is Debye fit.

provide an excellent description of the experimental data, using the following parameters: $:^{13} \epsilon_{\infty}=4.21, \epsilon_{s}=78.3$, $\tau=8.2 \times 10^{-12} \mathrm{~s}$ in the expressions

$$
\begin{aligned}
& \operatorname{Re} \epsilon=\epsilon_{\infty}+\frac{\epsilon_{s}-\epsilon_{\infty}}{1+\omega^{2} \tau^{2}}, \\
& \operatorname{Im} \epsilon=\frac{\left(\epsilon_{s}-\epsilon_{\infty}\right) \omega \tau}{1+\omega^{2} \tau^{2}} .
\end{aligned}
$$

In the resonance region there is no general formula to fit the experimental data. Here, a cubic spline interpolation ${ }^{16}$ is used to fit the experimental data and the integral is evaluated using this spline to interpolate the experimental results. For a thermal electron transfer only, frequencies below the electronic excitation region are relevant to our calculation. Thereby, the angular frequency $(\omega)$ region we consider is from 0 to $7.2 \times 10^{14} \mathrm{rad} / \mathrm{s}$, abbreviated in the following as $\mathrm{s}^{-1}\left(3844 \mathrm{~cm}^{-1}\right)$, since from $7.2 \times 10^{14} \mathrm{~s}^{-1}$ to the optical frequency the imaginary part of dielectric constant is extremely small ${ }^{15}$ (cf. Fig. 1). From 0 to $1.0 \times 10^{11}$ $\mathrm{s}^{-1}$ the Debye formula is used and from $1.0 \times 10^{11}$ to 7.2 $\times 10^{14} \mathrm{~s}^{-1}$ the spline interpolation is employed. The experimental data and the fitted results are collected in Fig. 1.

The inner contribution data is well-known from the literature, ${ }^{10,11} \omega_{1}=390 \mathrm{~cm}^{-1}, \omega_{2}=490 \mathrm{~cm}^{-1}, d_{0}=0.14 \AA$, $m=3.0 \times 10^{-26} \mathrm{~kg} /$ molecule.

The results of these calculations are given in Table $I$. Comparing the values for the quantum rate constant it is clcar that the saddlepoint approximation is very good, and the following discussion is based on that approximation. This quantum effect is seen in Table I to be substantially smaller than the simulation result from Chandler and coworkers ${ }^{6,7}$ who used the SPC model for water. This difference is due to the different spectral density employed. In their calculations the spectral density is obtained from the cosine transformation of the classical real time bath autocorrelation function which is calculated from the computer simulation. To illustrate this point we plot in Fig. 2 the

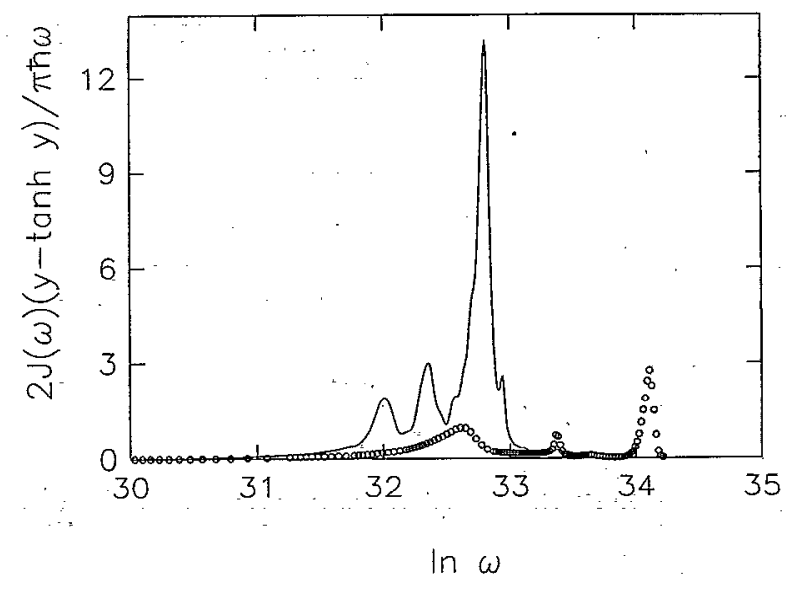

FIG. 2. The experimental data (Refs. 12-15) and the simulation result of the SPC model (Ref. 6). O denotes the integrand of experimental quantum correction factor from the outer part of the spectral density $J_{0}$, with $y=\beta \hbar \omega / 4$. The solid line is the integrand of SPC model quantum correction from the spectral density of Ref. 7, noting that the two small peaks around in $\omega=32$ arise from the inner part contribution.

integrand difference between the exact expression and the classical expression in the exponential part of Eq. (2.13) which gives most of the quantum correction factor (cf. Table I). In Fig. 2 the result is given for both the experimental outer spectral density and for the SPC water model, using in the latter case the results of Ref. 7. It should be remembered that the two small peaks around $\omega=1.0 \times 10^{14}$ $\mathrm{s}^{-1}$ are contributed by the inner part of the spectral density $J_{i}(\omega)$ since the curve is the total spectral density calculated from their simulation. From Fig. 2 we see that in one region (around $1.6 \times 10^{14} \mathrm{~s}^{-1}$ ) important for the quantum correction the SPC model considerably overestimates the dielectric response. In another region (around $6.5 \times 10^{14}$ $\mathrm{s}^{-1}$ ) important for quantum correction it has no contribution at all. Thus, we believe that the spectral density used in the SPC model is not accurate due to the rigid model of the solvent molecules employed-it contains neither the electronic nor the vibrational polarizability of the individual solvent molecules.

In order to test this supposition we have used two computer simulations available in the literature ${ }^{18}$ for the water, namely the TIP4P and the MCY models, both of which also omit the two molecular polarizabilities just mentioned. Although there exist some polarizable water models in the literature ${ }^{19}$ no detailed dielectric dispersion curve appears to be available from them. We have used the spectral density for the TIP4P and MCY models, with Eqs. (2.12) and (2.13), to calculate the quantum correction factor under the saddlepoint approximation. For the TIP4P model the outer spectral density $J_{0}(\omega)$ is calculated from a phenomenological formula, given in Ref. 18, which fits the simulation result well (Fig. 9 in Ref. 18), and the inner part, $J_{i}(\omega)$, is kept the same as above. The resulting quantum correction factor is 26 when the upper limit is $2.1 \times 10^{14} \mathrm{~s}^{-1}$, which is the valid limit of the phenomenological formula. For the MCY model the quantum correction factor is 21 when the upper limit is $2.2 \times 10^{14} \mathrm{~s}^{-1}$. 


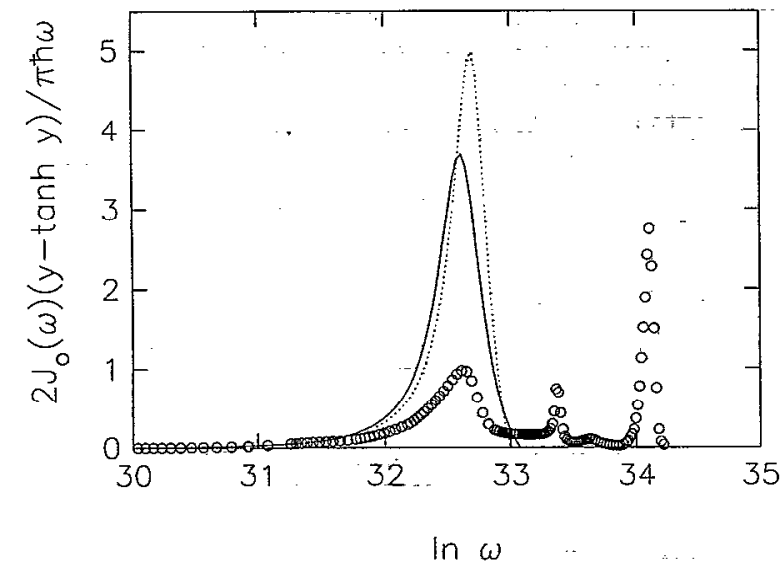

FIG. 3. The experimental data (Refs. 12-15) and the simulation result of the MCY and TIP4P models (Ref. 18). $O$ denotes the integrand of experimental quantum correction from the outer part spectral density $J_{0}$, with $y=\beta \hbar \omega / 4$. The solid line is the integrand of MCY model quantum correction from the outer part of the spectral density of Ref. 18. The dotted line is the integrand of the TIP4P model quantum correction from the outer part of the spectral density of Ref. 18.

These results can be understood from Fig. 3. Like the SPC model both the TIP4P and the MCY models overestimate the dielectric response in a region $(32 \leqslant \ln \omega \leqslant 33)$ and underestimate in another $(33 \leqslant \ln \omega \leqslant 34.3)$, regions which are critical for the quantum effect calculation. For the TIP4P and the MCY models the large spectral density is due to the small $\operatorname{Re} \epsilon$ of the model simulations in a critical region (Fig. 4).

For comparison with previous work, we give next an approximation to the outer contribution in Eq. (2.13) by dividing the complete frequency range of the dielectric response into two parts, a "classical" part and a "quantum" part, where a separation frequency $\omega_{\mathrm{cl}}$ is defined by relation $\beta \hbar \omega_{\mathrm{cl}} / 4=1.0$. From 0 to $\omega_{\mathrm{cl}}$ the classical approximation

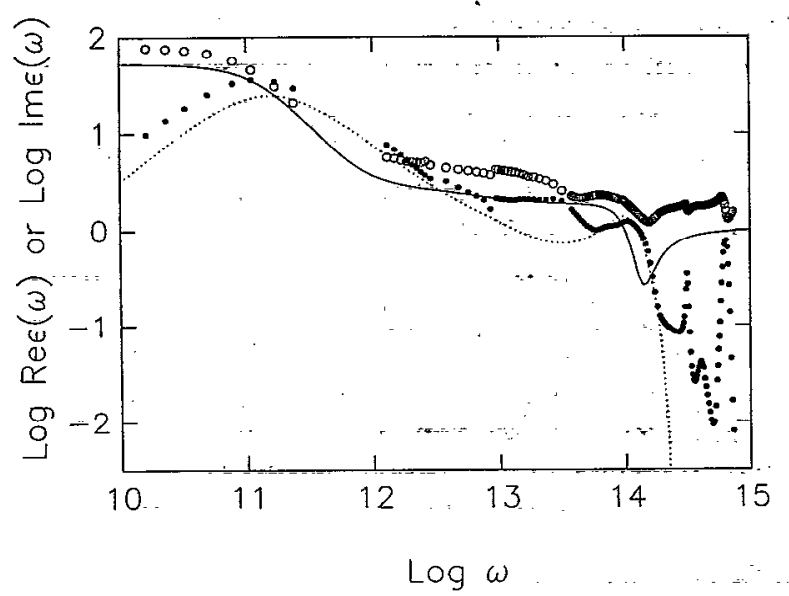

FIG. 4. The experimental data (Refs. 12-15) and the simulation result of the TIP4P model (Ref. 18). O denotes the real part of dielectric constant; the imaginary part. The solid line is the rcal part of the model simulation, and the dotted line is the imaginary part of the model simulation.

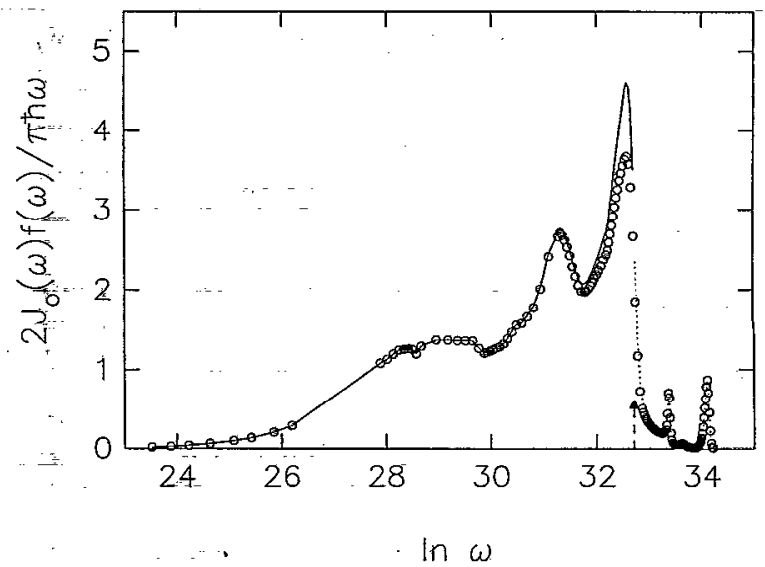

FIG. 5. The separation of classical modes and quantum modes approximation. $O$ denotes the integrand of experimental data from the outer part of the spectral density $[f(\omega)=\tanh (\beta \hbar \omega / 4)]$; the solid curve is the classical integrand [ $f(\omega)=\beta \hbar \omega / 4]$ from the experimental data; and the dotted curve the quantum integrand $[f(\omega)=1.0]$ from the experimental data. The arrow mark gives the separation frequency with $\beta \hbar \omega / 4=1.0$.

$(\beta \hbar \omega / 4<1.0)$ is used for the first part of the exponential factor in Eq. (2.13), written as $\exp \left[-\beta \lambda_{0}^{(1)} / 4\right]$, where

$$
\lambda_{0}^{(1)}=-\frac{\int\left|D^{f}-D^{i}\right|^{2} d \mathbf{r}}{4 \pi^{2}} \int_{0}^{\omega_{\mathrm{cl}}} d \omega \frac{\operatorname{Im} \epsilon(\omega)}{\omega|\epsilon(\omega)|^{2}} .
$$

By a sum rule, ${ }^{3}$ we have

$$
\int_{0}^{\omega_{\mathrm{cl}}} d \omega \frac{2}{\pi} \frac{\operatorname{Im} \epsilon(\omega)}{\omega|\epsilon(\omega)|^{2}}=\frac{1}{\epsilon\left(\omega_{\mathrm{cl}}\right)}-\frac{1}{\epsilon_{s}} .
$$

Equations (3.2) and (3.3) give the well-known classical type of expression for reorganization energy arising from this portion of the outer contribution. From $\omega_{\mathrm{cl}}$ to $\omega_{\mathrm{op}}$ the quantum limit gives $\tanh (\beta \hbar \omega / 4) \approx 1$, and that contribution to the exponential factor in Eq. (2.13) can then be written as

$$
\sigma=\exp \left[-\frac{2}{\pi \hbar} \frac{\int\left|D^{f}-D^{i}\right|^{2} d \mathbf{r}}{8 \pi} \int_{\omega_{\mathrm{cl}}}^{\omega_{\mathrm{op}}} d \omega \frac{\operatorname{Im} \epsilon(\omega)}{\omega^{2}|\epsilon(\omega)|^{2}}\right] .
$$

This latter factor is temperature independent and produces the tunneling factor arising from the quantum modes. A similar discussion can be found in Ref. 20 . In general, the quantum modes renormalize the coupling matrix. ${ }^{8}$ This limiting situation of dividing the modes into quantum and classical modes yields a fairly good approximation as seen in Fig. 5, the rate calculated from this approximation is smaller by a factor of 2 . These two types of modes tend to play different roles in the electron transfer, the former giving a nuclear tunneling effect and the latter generating an activation barrier, an effect which has been often discussed in the literature. ${ }^{1,2,3}$ From the tunneling factor expression (3.4), it is clear the really high frequency modes (say, higher than $7.2 \times 10^{14} \mathrm{~s}^{-1}$ ) do not make a significant contribution to the tunneling effect due to the negligible imaginary part of the dielectric constant. In this sense the electronic polarization does not make large contribution to the 
electron transfer rate. However, the electronic polarization, by creating a shielding effect, does influence the other aspect of the $\epsilon(\omega)$ behavior. Furthermore, the atomic polarization (in the vibrational resonance region) does contribute to the electron transfer, both directly and via shielding, indirectly.

\section{CONCLUDING REMARKS}

One of the particular features of the present work is to illustrate the calculation of nonadiabatic electron transfer rate from the experimentally available data using the linear response approximation and to test certain solvent molccular models (SPC, TIP4P, and MCY) in the literature. For aqueous ferrous-ferric system, the calculated rate from the experimental data is near the traditional estimate, ${ }^{4,5}$ but different from a recent pioneering molecular simulation result of Chandler and co-workers. Even where the latter is approximated by introducing a harmonic bath approximation a significant difference remains. It will be interesting to repeat the molecular simulation using a molecular model of liquid water which includes both the atomic and electronic polarization and gives the correct dielectric dispersion behavior of water, rather than mainly the static dielectric constant.

\section{ACKNOWLEDGMENTS}

We would like to thank Dr. A. A. Stuchebrukhov and Dr. D. Chandler for insightful discussions. We are indebted to Dr. Joel Bader for providing the data file used in Fig. 2. It is a pleasure to acknowledge, too, the support of this research by the National Science Foundation and the Office of Naval Research.

${ }^{1}$ See, e.g., R. A. Marcus and N. Sutin, Biochem. Biophys. Acta 811, 265 (1985).
${ }^{2}$ (a) V. G. Levich and R. R. Dogonadze, Dokl. Acad. Nauk. SSSR 124, 123 (1959); (b) R. R. Dogonadze, A. M. Kuznetsov, and T. A. M. Marsagishvili, Electrochim. Acta 25, 1 (1980); (c) A. A. Ovchinnikov and M. Ya. Ovchinnikova, Sov. Phys. JETP 29, 688 (1969).

${ }^{3}$ J. Ulstrup, Charge Transfer in Condensed Media (Springer, Berlin, 1979).

${ }^{4}$ See, for example, B. S. Brunschwig, J. Logan, M. D. Newton, and N. Sutin, J. Am. Chem. Soc. 102, 5798 (1980).

${ }^{5}$ (a) R. A. Marcus, J. Chem. Phys. 43, 679 (1965); (b) P. Siders and R. A. Marcus, J. Am. Chem. Soc. 103, 741 (1981).

${ }^{6}$ R. A. Kuharski, J. S. Bader, D. Chandler, M. Spirk, M. L. Klein, and R. W. Impey, J. Chem. Phys. 89, 3248 (1988); J. S. Bader, R. A. Kuharski, and D. Chandler, ibid. 93, 230 (1990); the term "spin" is conventionally used since the two electronic states are taken as two states of a pseudospin variable, and "boson" comes from that the harmonic bath follows the Bose-Einstein statistics.

${ }^{7}$ J. S. Bader, Ph.D. thesis, University of California, Berkeley, 1990.

${ }^{8}$ X. Song and A. A. Stuchebrukhov, J. Chem. Phys. 99, 969 (1993).

${ }^{9}$ D. Chandler, in Les Houches, Session LI, 1989, Part I, Liquids, Freezing and the Glass Transition, edited by D. Levesque, J. P. Hansen, and J. Zinn-Justin (Elsevier, New York, 1991).

${ }^{10}$ B. S. Brunschwig, J. Logan, M. D. Newton, and N. Sutin, J. Am. Chem. Soc. 102, 5798 (1980).

${ }^{11}$ N. Sutin, Prog. Inorg. Chem. 30, 441 (1983).

${ }^{12}$ J. B. Hasted, Aqueous Dielectrics (Chapman and Hall, London, 1973).

${ }^{13}$ M. N. Afsar and J. B. Hasted, Infrared Phys. 18, 835 (1978).

${ }^{14}$ J. B. Hasted, S. K. Husain, F. A. M. Frescura, and J. R. Birch, Infrared Phys. 27, 11 (1987).

${ }^{15}$ G. M. Hale and M. R. Querry, Appl. Opt. 12, 555 (1973).

${ }^{16}$ W. H. Press, B. P. Flannery, S. A. Teukolsky, and W. T. Vetterling, Numerical Recipes (Cambridge Universily, Cambridge, 1989).

${ }^{17}$ Here, we use a two-sphere model which neglects dielectric image effects for calculational convenience. The quantum correction factor is not expected to change significantly for a different model of ions.

${ }^{18}$ M. Neumann, J. Chem. Phys. 85, 1567 (1986).

${ }^{19}$ See, e.g., K. Watanabe and M. L. Klein, Chem. Phys. 131, 157 (1989); P. Madden and D. Kivelson, Adv. Chem. Phys. 56, 467 (1984); S. B. Zhu, S. Yao, J. B. Zhu, S. Singh, and G. W. Robinson, J. Phys. Chem. 95, 6211 (1991).

${ }^{20}$ M. Marchi, J. N. Gehlen, D. Chandler, and M. Newton, J. Am. Chem. Soc. 115, 4178 (1993). The Appendix of this paper also comments on the role of electronic polarizability in scaling down the magnitude of the reorganization energy. 